Chapter 2, section 1 in: Developments in Laser Techniques and Fluid Mechanics, Eds. Adrian, Durao, Durst, Heitor, Maeda, and Whitelaw; Springer-Verlag, 1997.

Presented as a Plenary Session at the 8th International Symposium on Applications of Laser Techniques to Fluid Mechanics; Lisbon, Portugal; July 8-11, 1996; pp. 1.2.1 - 1.2.12 in the conference proceedings.

\title{
Molecular Tagging Diagnostics for the Study of Kinematics and Mixing in Liquid-Phase Flows
}

\author{
M. M. Koochesfahani ${ }^{\mathrm{a}}$, R. K. Cohn ${ }^{\mathrm{a}}$, C. P. Gendrich ${ }^{\mathrm{a}}$, and D. G. Nocera ${ }^{\mathrm{b}}$ \\ Departments of Mechanical Engineering ${ }^{\mathrm{a}}$ and Chemistry ${ }^{\mathrm{b}}$ \\ Michigan State University, East Lansing, MI 48824
}

\begin{abstract}
This work reports our recent developments of novel techniques for velocimetry and studies of the Lagrangian evolution of mixing interfaces based on molecular tagging approaches. These developments take advantage of a class of newly engineered phosphorescent supramolecules that are water soluble. Previous implementations using photochromic molecules and caged fluorescein are briefly discussed and compared. The application of molecular tagging velocimetry is demonstrated in several flow fields, including examples which illustrate its capability in flows with significant out-of-plane motion and its potential for simultaneous passive scalar and velocity measurements.
\end{abstract}

Keywords. molecular tagging, optical diagnostics, supramolecules, velocimetry

\section{Introduction}

The capability to tag a portion of a flow non-intrusively and observe its subsequent evolution offers new possibilities for velocimetry and the study of entrainment and mixing in fluid flows. Chemical compounds with long luminescence lifetimes are needed for this approach. Current laser induced fluorescence (LIF) techniques are generally not suitable for this purpose because of the short lifetime of the fluorescence process ( $\tau=$ a few nanoseconds).

The majority of past efforts in the use of molecular tagging have concentrated on flow velocimetry. Regardless of the details of the photophysics in each molecular design, all of these efforts can be characterized under a broader common heading of Molecular Tagging Velocimetry (MTV). In this method of velocimetry, the flowing medium is premixed with molecules that can be turned into long lifetime tracers upon excitation by photons. Typically a pulsed laser is used to "tag" small regions of interest. The tagged regions are imaged at two successive times within the lifetime of the tracer. The measured displacement vector provides the estimate of the velocity vector. 
Past implementations of MTV in liquid phase flows primarily include the use of photochromic molecules (Popovich \& Hummel 1967; Falco \& Chu 1987; Ojha, et al. 1989; and Chu \& Liao 1992, among others), caged fluorescein (Lempert, et al. 1995; Harris, et al. 1996a \& 1996b; Biage, et al. 1996), and specially engineered phosphorescent supramolecules (Koochesfahani, et al. 1993; Stier, 1994; Gendrich, et al. 1994; Hill \& Klewicki, 1995, Cohn, et al. 1995, and Gendrich \& Koochesfahani 1996). In gas phase flows, the fluorescence of excited-state oxygen (Miles, et al. 1987; 1989; 1993) and the phosphorescence of biacetyl (Hilbert \& Falco 1991, Stier, et al. 1995) have typically been utilized. The review by Falco \& Nocera (1993) discusses some of these and other efforts known at that time.

The purpose of this work is to give a brief summary of various MTV implementations and their chemical mechanisms, followed by our recent contributions to improve and extend this technique. This includes a discussion of the ideas behind the design of the new phosphorescent complexes and our approach to imaging and tracking the tagged regions. Examples of the application of MTV to different flow fields will be provided, one of which highlights the ability to make measurements when strong out-of-plane motions are present. Other examples will illustrate the potential for simultaneous passive scalar and velocity measurements.

\section{Chemical Mechanisms}

A molecular complex is suitable for molecular tagging applications if its lifetime as a tracer is long enough relative to the flow convection time scale to allow sufficient displacement of the tagged regions. The discussion in this work focuses primarily on liquid phase applications, however it is worthwhile to briefly note some highlights of work in gas phase. The use of excited-state oxygen fluorescence, pioneered by Miles, et al. $(1987,1989,1993)$ under the acronym RELIEF, is the only tagging method currently available in an oxygen environment (e.g. air). The lifetime of the tracer (vibrationally excited $\mathrm{O}_{2}$ ) is of order $100 \mu \mathrm{s}$, making it suitable for high speed flows. The phosphorescence of biacetyl, which has a reported lifetime of order $1 \mathrm{~ms}$, has also been used for velocimetry (Hilbert \& Falco 1991, Stier, et al. 1995). However, its use is limited to oxygen-free environments due to the phosphorescence quenching by oxygen.

\subsection{Photochromics}

In a photochromic process a molecule $\mathrm{M}$ is excited to produce a high energy form of $\mathrm{M}$, designated $\mathrm{M}^{\prime}$, which has a different absorption spectrum giving rise to a color change (e.g. from clear to dark blue). The long lifetime tracer is the newly produced $\mathrm{M}^{\prime}$, which persists for several seconds to minutes. The nonradiative conversion from $\mathrm{M}$ to $\mathrm{M}^{\prime}$, i.e. the tagging process, occurs rapidly (within the duration of a few nanosecond long laser pulse). The photochromic process is reversible; $\mathrm{M}^{\prime}$ thermally converts back to $\mathrm{M}$ over time, therefore the chemical is reusable. Photochromic dyes are generally insoluble in water, so organic liquids such as kerosene 
are typically used as the flowing medium. The use of photochromic chemicals requires two photon sources; typically a UV laser (e.g. $\lambda=351 \mathrm{~nm}$ from an excimer laser) to induce the color change and a white light source to interrogate the tagged regions.

Hummel and his group (e.g. Popovich \& Hummel 1967, Ojha, et al. 1989) originated the use of photochromic chemicals as a velocity measurement tool by tagging the flow along single lines. Significant improvements were made in the pioneering work of Falco \& Chu (1987), who used a laser grid to tag the flow and coined the acronym LIPA (Laser Induced Photochemical Anemometry). The distinction between these different tagging approaches is a very important one, which will be discussed in Section 3.

Some of the advantages of photochromics (long lifetime, reusable) are offset by the need to use special fluids such as kerosene. It is possible to ease this restriction by making chemical modifications to enable many photochromic dyes to dissolve in water. Such examples have been reported by Yurechko \& Ryazantsev (1991), and Douglas, et al. (1991). The most significant drawback in using photochromic chemicals is that the image is produced by a change in absorbence, thereby requiring a measurement of the difference between incident and transmitted light. Emitted light (against a black background) is more easily and accurately detected than transmitted light; consequently, images based on luminescence are better suited to MTV applications. Despite some of these difficulties, photochromics are being used very effectively to advance the understanding of flow physics (Chu \& Liao, 1992; Chu, et al. 1993).

\subsection{Caged Fluorescein}

In this compound a chemical group is attached to fluorescein in order to render it non-fluorescent. The caging group is removed upon absorption of UV photons ( $\lambda$ $\cong 350 \mathrm{~nm}$ ), thereby creating regular fluorescein which fluoresces with very high quantum efficiency. In this case, the long lifetime tracer is the uncaged fluorescein, which persists for a very long time and can be interrogated at the time of interest through its luminescence upon re-irradiation. Two sources of photons are therefore needed, one to break the cage and the other to excite fluorescence. The use of caged fluorescein and similar compounds for molecular tagging velocimetry was first introduced by Lempert, et al. (1995) under the acronym PHANTOMM (PHoto-Activated Non-intrusive Tracking of Molecular Motion). In both this work and several recent novel applications of it (Harris, et al. 1996a \& 1996b; Biage, et al. 1996), laser line tagging is used (see Section 3 for further discussion).

There are two aspects of caged fluorescein that one must be aware of in designing an experiment. The cage-breaking process is irreversible; each caged molecule can be used only once. The cage-breaking process is not rapid and occurs with a time constant of the order of a few milliseconds. This delay between laser tagging and generation of enough fluorescein to obtain an image with sufficient signal/ noise will dictate the fastest flow speeds that can be accommodated. Gendrich, et al. (1996) have recently presented a technique for overcoming this problem based 
on a two-detector approach. On the other hand, very slow speed flows can be handled with ease considering the very long lifetime (practically infinite) of the uncaged fluorescein. It is anticipated that future research will lead to the design of new generations of caged fluorescent molecules without these drawbacks.

\subsection{Phosphorescent Supramolecules}

In using a phosphorescent compound for molecular tagging, excitation by photons is used to produce a long-lived excited state which is interrogated through its phosphorescence emission as it radiatively returns to its ground state. The long lifetime tracer is the excited state molecule itself. In this case only one source of photons is needed, and the excitation/emission process is reversible. The difficulty is that the long-lived excited state (i.e. phosphorescence) suffers from $\mathrm{O}_{2}$ and $\mathrm{H}_{2} \mathrm{O}$ quenching, and as a result, suitable molecular complexes have not been available until recently.

New findings by Nocera and his group (Ponce, et al. 1993, Mortellaro \& Nocera 1996, Hartmann, et al. 1996) show that supramolecules may be designed that exhibit long-lived phosphorescence, which is not quenched. A successful design by Ponce, et al. (1993) indicates that the quenching of a lumophore can be stopped, and the phosphorescence emission recovered, by mixing certain alcohols with an aqueous solution of a cyclodextrin (CD) cup that contains the lumophore. Cyclodextrins are molecules constructed from sugars connected in a head-to-tail arrangement. The molecule is cup-shaped with its size determined by the number of sugars in the structure. The $\mathrm{CD}$ used in our application is GB-CD, which is constructed of 7 glucose subunits, resulting in an outer cup dimension of $15.3 \AA$ and an inner cup cavity dimension of $7.8 \AA$. The size of the cavity is important for effective binding to the guest molecule, in our case 1-bromonaphthalene (1-BrNp), which is the lumophore.

The long-lived, green phosphorescence (lifetime $\tau \approx 5 \mathrm{~ms}$ ) of 1-BrNp is efficiently quenched by oxygen, even when the lumophore is inside a CD cup. The phosphorescence can be recovered upon adding an alcohol (ROH) to the solution as shown in Figure 1. Detailed studies of Ponce, et al. (1993) and Hartmann, et al. (1996) show that a ternary complex $(1-\mathrm{BrNp} \cdot \mathrm{GB}-\mathrm{CD} \cdot \mathrm{ROH})$ is formed, where the alcohol hydrogen bonds to the rim of the $\mathrm{CD}$ cup and acts as its lid, thereby shielding 1-BrNp from oxygen. The phosphorescence enhancement can be very large, approaching $10^{4}-10^{5}$, depending on the fit of the alcohol lid to the CD cup. For the studies described in this work, cyclohexanol was used as the alcohol.

When using this phosphorescent complex, it is useful to recall that luminescence lifetime refers to the time when the emission has decayed to $37 \%\left(\mathrm{e}^{-1}\right)$ of its initial intensity. The actual usable delay time between laser tagging and interrogation can be considerably longer and is dictated by the type of detection used. We have, for example, measured flows with delays of up to $30 \mathrm{~ms}$ using image-intensified cameras. Nevertheless, the lifetime of this molecular design is not suitable for very low speed flows. Such cases are best investigated using caged fluorescein or photochromics. 

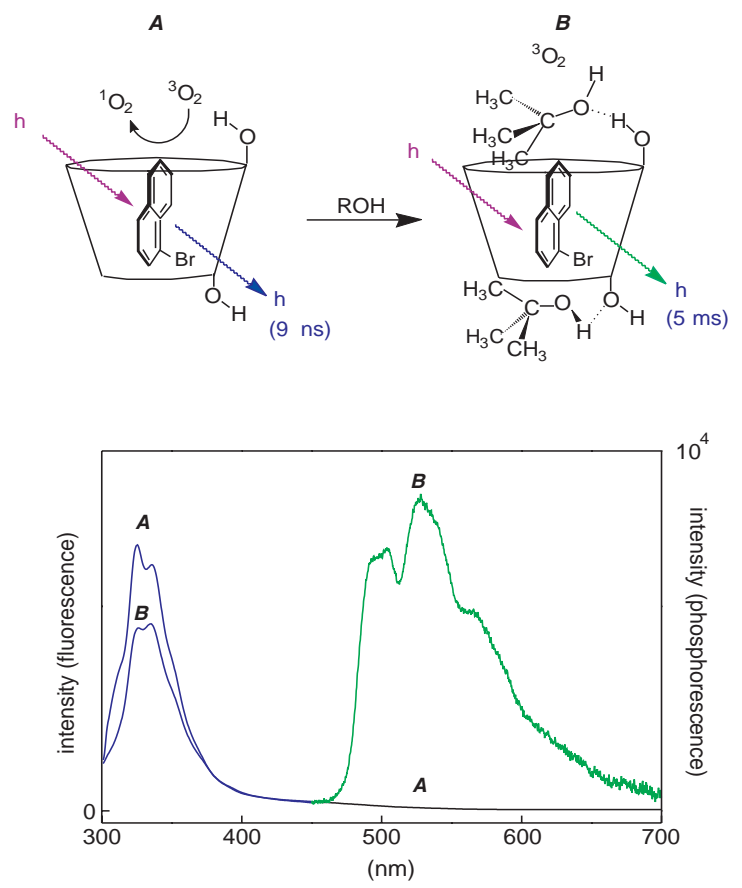

Fig. 1. Emission from 1-BrNp in G $\beta-C D$ (A) Only blue fluorescence is exhibited in the absence of an appropriate alcohol ( $\mathrm{ROH})$; (B) A bright green phosphorescence plus the initial fluorescence is seen upon the addition of $\mathrm{ROH}$, which prevents the quenching of 1-BrNp phosphorescence by $\mathrm{O}_{2}$.

Depending on the placement of the three components of the phosphorescent complex in the flow, we have devised methods for (1) purely velocimetry, (2) molecularly tagging a passive scalar mixing region and monitoring its Lagrangian evolution, and (3) molecularly tagging a chemical reaction interface between two streams and observing its Lagrangian evolution. The first two of these applications are described in this work. Details of the experimental implementations for all three applications can be found in Gendrich, et al. (1996).

\section{Tagging, Detection, and Processing Schemes}

\subsection{Tagging}

Tagging the flow along single lines was originally used by Hummel and his group (e.g. Popovich \& Hummel 1967, Ojha, et al. 1989). The velocity is determined from the displacement of the tagged lines in much the same manner as using hydrogen bubble lines generated by a wire (for example, see Lu \& Smith, 1985). Laser line tagging is still the only method used to date in the works utilizing caged fluo- 
rescein (Lempert, et al. 1995; Harris, et al. 1996 \& 1996b; Biage, et al. 1996) and excited-state oxygen fluorescence (Miles, et al. 1987, 1989, 1993). It is very important to recognize that line tagging allows the measurement of only one component of velocity, that normal to the tagged line. In addition, the estimate of this velocity component has an inherent error associated with it. Following the analysis of Hill \& Klewicki (1995), and referring to Figure 2, this error can be cast in the form

$$
\frac{\Delta u}{u}=\tan \theta \frac{\partial u}{\partial y} \Delta t
$$

In this expression, $u$ is the estimated velocity component normal to the tagged line, $\Delta u=u_{\text {actual }}-u$ is the error in the estimated velocity, $\theta$ is the local flow angle given by $\tan \theta=v / u$ with $v$ being the flow velocity parallel to the tagged line, and $\Delta t$ is the time delay between tagging and interrogation. Clearly an a priori knowledge of the flow field is necessary in order to provide an estimate of the error. It can be observed, however, that this inherent error is identically zero only in flows where the velocity component $v$ along the tagged line is zero (i.e. unidirectional flows) or where the velocity gradient $\partial u / \partial y=0$. In a general flow field where these constraints are not met, the error can be reduced by decreasing the delay time $\Delta t$, but it cannot be made arbitrarily small, since $\Delta t$ has to be large enough for the resulting displacement of the tagged line to be measured with adequate accuracy. While keeping these issues in mind, it is sometimes possible to take advantage of an a priori knowledge of the flow field under investigation to design the experimental parameters such that the inherent error discussed here becomes minimal compared to other measurement errors. In cases where this can be done (see Hill \& Klewicki 1995, for example) the line tagging approach can provide reliable information on one component of the velocity vector.

In order to measure two components of the velocity in a plane unambiguously, the luminescence intensity field from a tagged region must have spatial gradients along two, preferably orthogonal, directions. For single-point velocimetry, this is

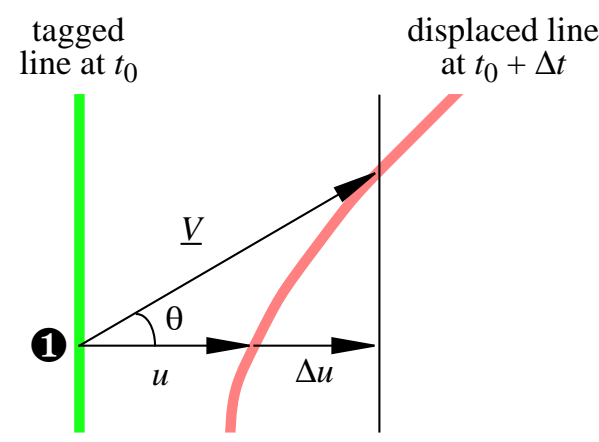

Fig. 2. The velocity at point (1) is $\underline{V}$. Using the line center displacement gives a lateral velocity estimate $u$, with error $\Delta u$. The vertical velocity component cannot be estimated with the flow tagged in this manner. 
easily achieved using a pair of crossing laser beams; a grid of intersecting laser lines allows multi-point velocity measurements. Use of this tagging scheme was first suggested by D'Arco, et al. (1982), and it was later improved upon and utilized by Falco \& Chu (1987). An example of a region in a flow tagged in this manner using our new phosphorescent supramolecules, and the same region interrogated subsequently at two different delay times, is depicted in Figure 3. The flow field is that generated by a vortex ring impacting a solid wall at normal incidence, which will be discussed as one of the examples in Section 4. The superposition of the image at the longer time delay (Figure 3c) onto the initially tagged image (Figure 3a), shown in Figure 4, serves to highlight the velocity error inherent in the line tagging approach discussed earlier. The image at the shorter time delay in Figure $3 \mathrm{~b}$ is more typical of that used in our velocimetry applications.

As will be seen later, laser grid tagging is only a special case of a more generalized approach to induce a spatially non-uniform luminescence intensity in a tagged region. For example, the non-uniform passive scalar concentration field typical of most turbulent flows can sometimes be used as a natural source of luminescence non-uniformity without the need for grid illumination.

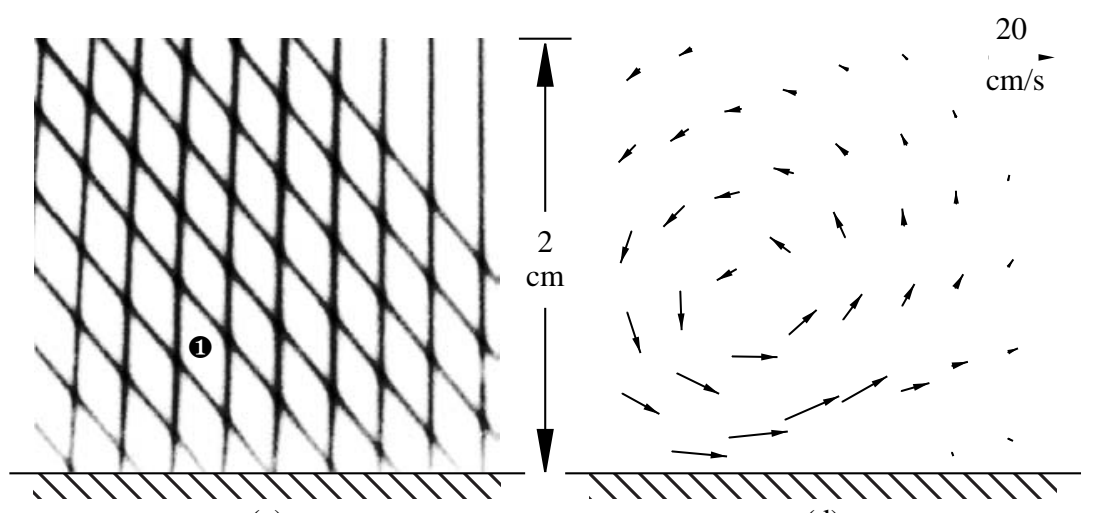

(a)

(d)

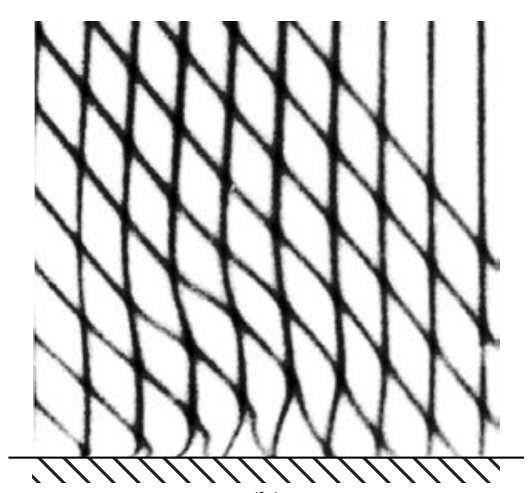

(b)

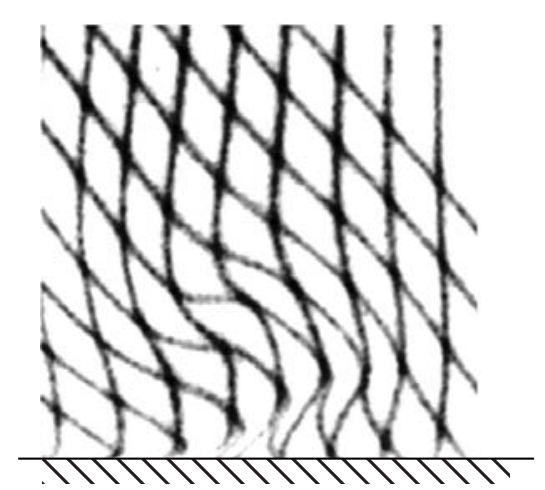

(c)

Fig. 3. (a) Undistorted grid. Distorted grid after two different delays: (b) $\Delta \mathrm{t}=5 \mathrm{~ms}$. (c) $\Delta \mathrm{t}$ $=19 \mathrm{~ms}$. (d) The velocity field resulting from correlating (a) with (b). 


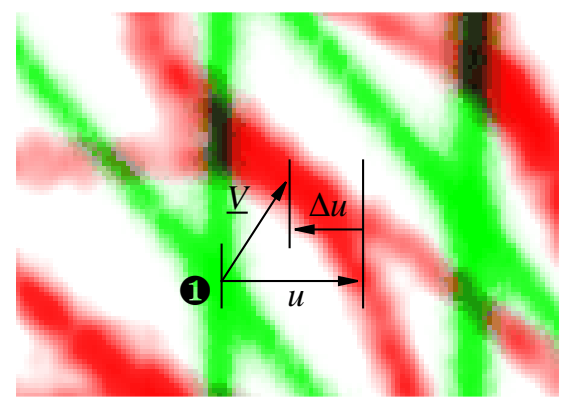

Fig. 4. The superposition of Figure 3c (red) over Figure 3a (green) in the neighborhood of the point marked 1 in Figure 3a. The notation is the same as in Figure 2.

\subsection{Detection}

Depending on the specific requirements of a particular application, a variety of image acquisition methods have been used in the past including cameras recording on film, CCD cameras, and gated image-intensified cameras. The common element among all previous studies is that a single detector is used; the initial tagging pattern is recorded once, usually at the beginning of the experiment, and then the delayed images are acquired. The implicit assumption in this approach is that the initial tagging pattern remains spatially invariant throughout the experiment. Because current processing schemes (see Section 3.3) measure the displacement of the tagged regions with sub-pixel accuracy, small variations in the initial pattern will be misinterpreted as flow velocity fluctuations. We have improved the accuracy of the MTV technique by employing a two-detector imaging scheme.

The experimental arrangement, shown in Figure 5, involves a link between the pulsed excimer laser $(\lambda=308 \mathrm{~nm})$ and two image detectors through a digital delay generator. Immediately after the laser fires, the first detector records an image of the tagged flow, and after a prescribed time delay $\Delta t$, the second detector records a delayed image. These images are digitally acquired in real time by two separate acquisition systems. The advantage of this arrangement is that any spatial "wandering" of the tagged regions (e.g. due to laser beam pointing instability, vibration of the optics, etc.) does not contribute to error in the measurement of the displacement of these regions. Furthermore, the two-detector setup is clearly indispensable when the intensity field in the initial tagging pattern cannot be assumed, for example in the case of a non-uniform scalar mixing field.

The data shown in this work were acquired using the arrangement shown in Figure 5 in conjunction with a variety of CCD detectors (interlaced cameras, electronically-shuttered frame transfer cameras, and gated image-intensified cameras) depending on the optical/imaging requirements unique to the particular experiment. Each image was digitized to 8 bits into a 512 × 512 pixel array. Time series data were acquired at a rate of 30 image pairs per second directly onto a 10-GB hard disk array. 


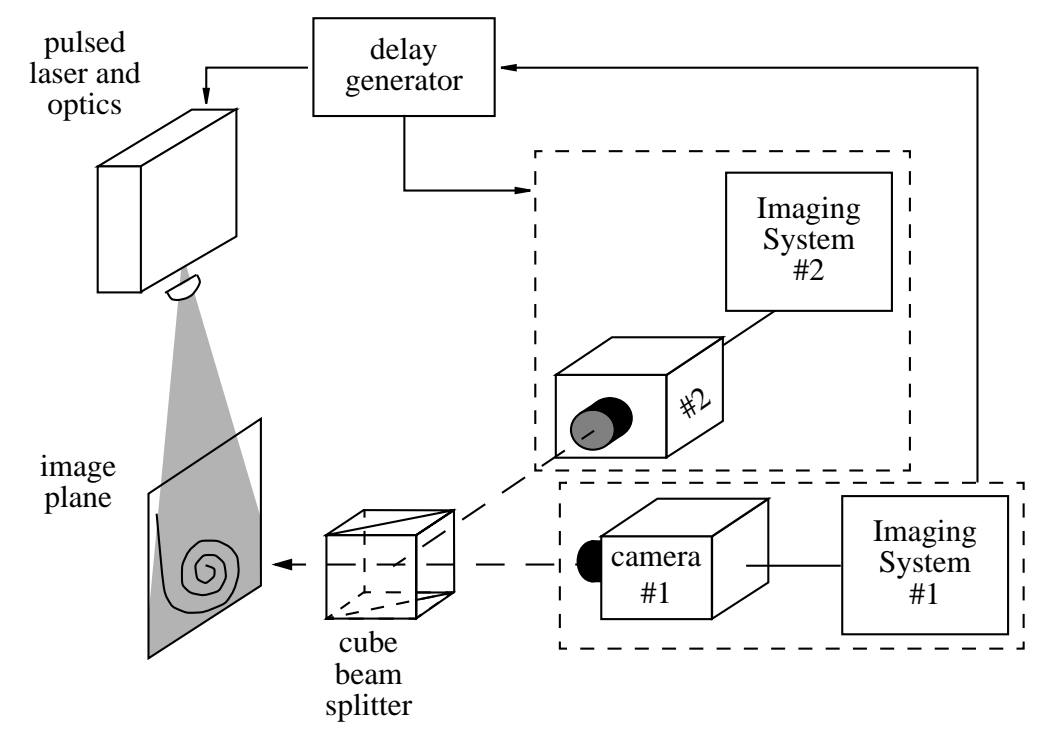

Fig. 5. Optical and electronic arrangement for 2-camera MTV experiments. Both cameras view the same image plane through the cube beam splitter. Synchronization between the two cameras and laser is provided by a digital delay generator.

\subsection{Processing}

The traditional method for finding the displacement of tagged lines or grids has been to locate the center of each line through various techniques. Most of the recent techniques use the best fit to an assumed laser line shape, for example, a gaussian intensity distribution. We are not aware of a systematic statistical study of the performance of this approach while considering the effects of experimental parameters such as image contrast, signal to noise ratio, etc. The recent study of Hill \& Klewicki (1995) reports the accuracy in determining the displacement vector to be \pm 0.35 pixel rms. Even though efforts are under way to improve the accuracy further using better algorithms, the basic premise behind this approach is the use of a known line shape for the tagged line, which may not be known a priori in some situations, due to a variety of reasons associated with laser beam transmission through a flowing medium, bleaching effects, etc.

We have taken a different approach in an attempt to implement a generalized scheme that is independent of the details within a tagged region, and can accommodate arbitrary tagging patterns including those due to non-uniform scalar mixing fields. The displacement of the tagged regions is determined using a direct digital spatial correlation technique. The example provided in Figure 6 illustrates a region in an actual experiment tagged by a laser grid, the tagged region at a later time, and the corresponding spatial correlation coefficient field for one of the grid crossings. A well-defined correlation peak occurs at the location corresponding to the displacement of the tagged region by the flow; the displacement peak is located to sub- 


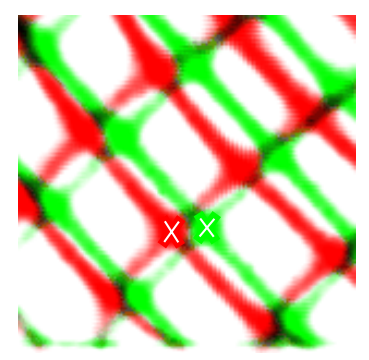

(a)

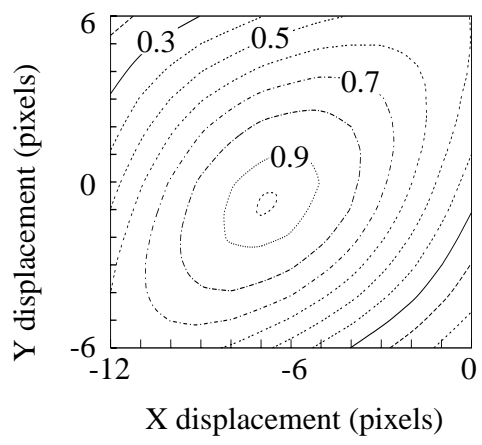

(b)

Fig. 6. Experimental MTV grid. (a) $\square$ lines are the grid at $t=0$; $\square$ lines are the grid after a $6 \mathrm{~ms}$ delay. (b) Correlation coefficient contours for the indicated intersection in (a)

pixel accuracy using a multi-dimensional polynomial fit. This procedure is similar to what could be used in DPIV processing of particle image pairs. One advantage of our processing technique over traditional line-center methods is robustness to the presence of noise due to the averaging process inherent in the correlation procedure. Based on both experiments and an extensive statistical study on the performance of this correlation approach, we have found that we can typically measure the displacement of the tagged regions with a $95 \%$ confidence limit of \pm 0.1 subpixel accuracy (i.e. $95 \%$ of the displacement measurements are accurate to better than 0.1 pixel). This corresponds to an rms accuracy of \pm 0.05 pixel, assuming a Gaussian distribution for error. The details can be found in Gendrich \& Koochesfahani (1996).

It should be noted that the spatial correlation method just described, though of more general utility than the line-center methods, may lead to a somewhat degraded spatial resolution. The contrast is analogous to that between PIV (using a group of seed particles) versus individual particle tracking.

\section{Examples of MTV Measurements}

When the three components of the phosphorescent complex (1-BrNp - Gß-CD $\mathrm{ROH})$ are premixed with water in the entire flow facility, we obtain a technique intended solely for velocity measurements. Since the flowing medium is homogeneously mixed, a pulsed laser grid pattern is used to molecularly tag the regions of interest.

An example of the type of time series data obtained from a single MTV grid intersection is shown in Figure 7. This figure illustrates the time evolution of two components of the velocity vector at a point near the axis of symmetry of a passing vortex ring. We note that this represents raw data (i.e. no filtering or smoothing) from one of many grid points where simultaneous velocity data were obtained. For 
an ideal vortex ring, at this location in the flow the v-component is expected to remain at zero and the u-component to smoothly increase to a maximum and subsequently drop to zero as the ring passes by. This behavior is captured by the data in Figure 7.

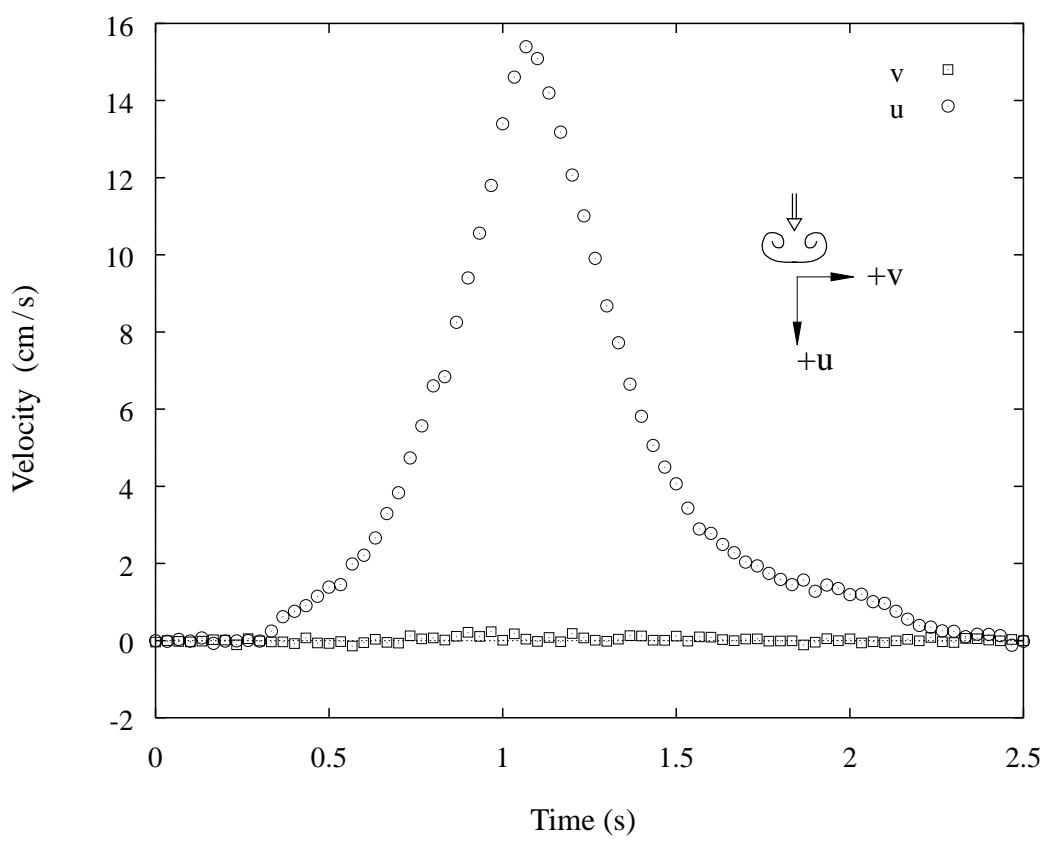

Fig. 7. The time evolution of (u,v) velocity components measured by MTV near the center of a passing vortex ring.

The next example provides a sample of the instantaneous whole-field measurements of two components of the velocity vector over a plane in a study of unsteady boundary layer separation in a vortex-wall interaction. A vortex ring approaching a wall at normal incidence generates an unsteady adverse pressure gradient on the wall, which results in boundary layer separation and the formation of a secondary vortex. An LIF visualization of this flow right after the formation of the secondary vortex is depicted in Figure 8(a) (only the left half of the flow is included). Figure 8(b) shows the corresponding instantaneous MTV velocity data (1 mm grid spacing with first grid $0.5 \mathrm{~mm}$ away from the wall) and the computed vorticity field. The data sequence in Figure 9 is selected from a much longer time series showing an enlarged view of the details of the velocity and vorticity fields during the boundary layer separation process. Except for the inherent smoothing involved in mapping the velocity data from the irregular MTV grid onto a regular grid, the data shown in Figures 8 and 9 represent raw data, i.e. no attempt was made to remove a "bad" vector, replace it, or apply spatial filtering. 


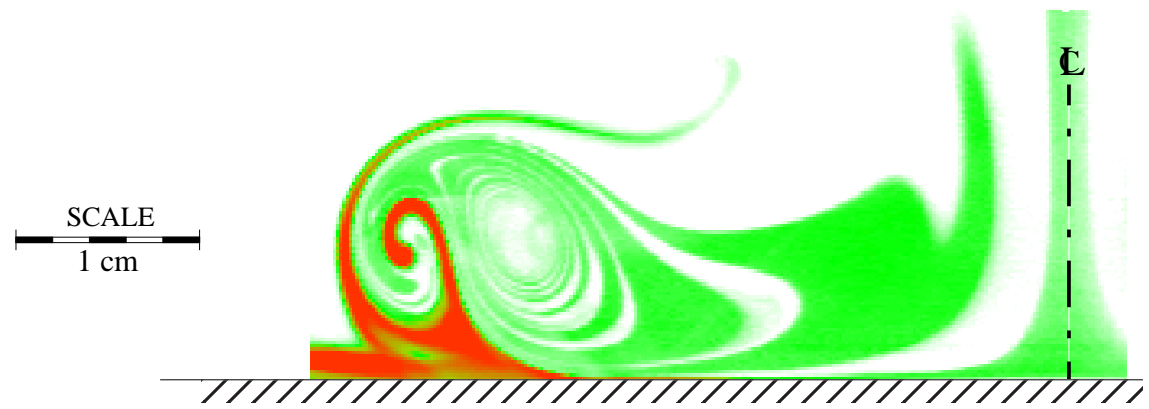

(a)

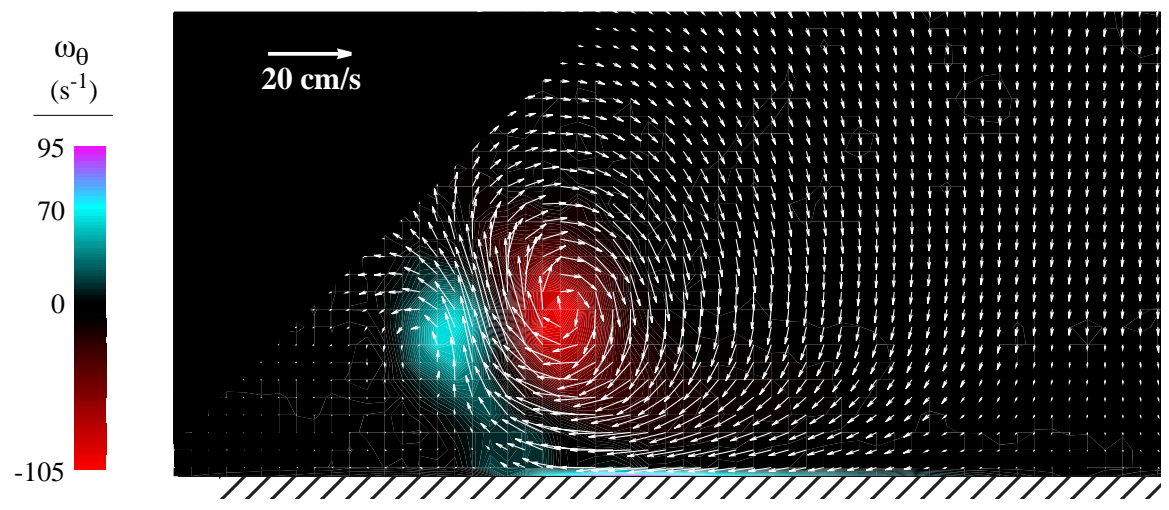

(b)

Fig. 8. (a) LIF image of the left-half of a downward moving vortex ring. The ring and wall-layer fluids are marked by green- and red-emitting laser dyes (the light grey and dark grey colors above), respectively. (b) Velocity and vorticity fields measured using MTV.

At this stage, a comparison between the MTV and PIV methods is warranted. The MTV technique can be thought of as the molecular version of PIV with two major advantages. First, since particles are replaced by molecules dissolved in the flowing medium, the problems related to particles tracking the flow, density mismatch between particles and the fluid, particle seeding density, etc. are all absent from MTV. Second, the performance and accuracy of PIV in measuring the inplane velocity vector can degrade in highly 3-D velocity fields due to particle motion in/out of the plane of the laser sheet. In MTV, the measurement of in-plane velocity vectors is quite insensitive to the out-of-plane velocity component. The reason is that only the tagged region is luminescent; the motion of any molecules located outside of this tagged region into or out of the image field of view does not contaminate the spatial correlation process, since those molecules are not luminescent. 

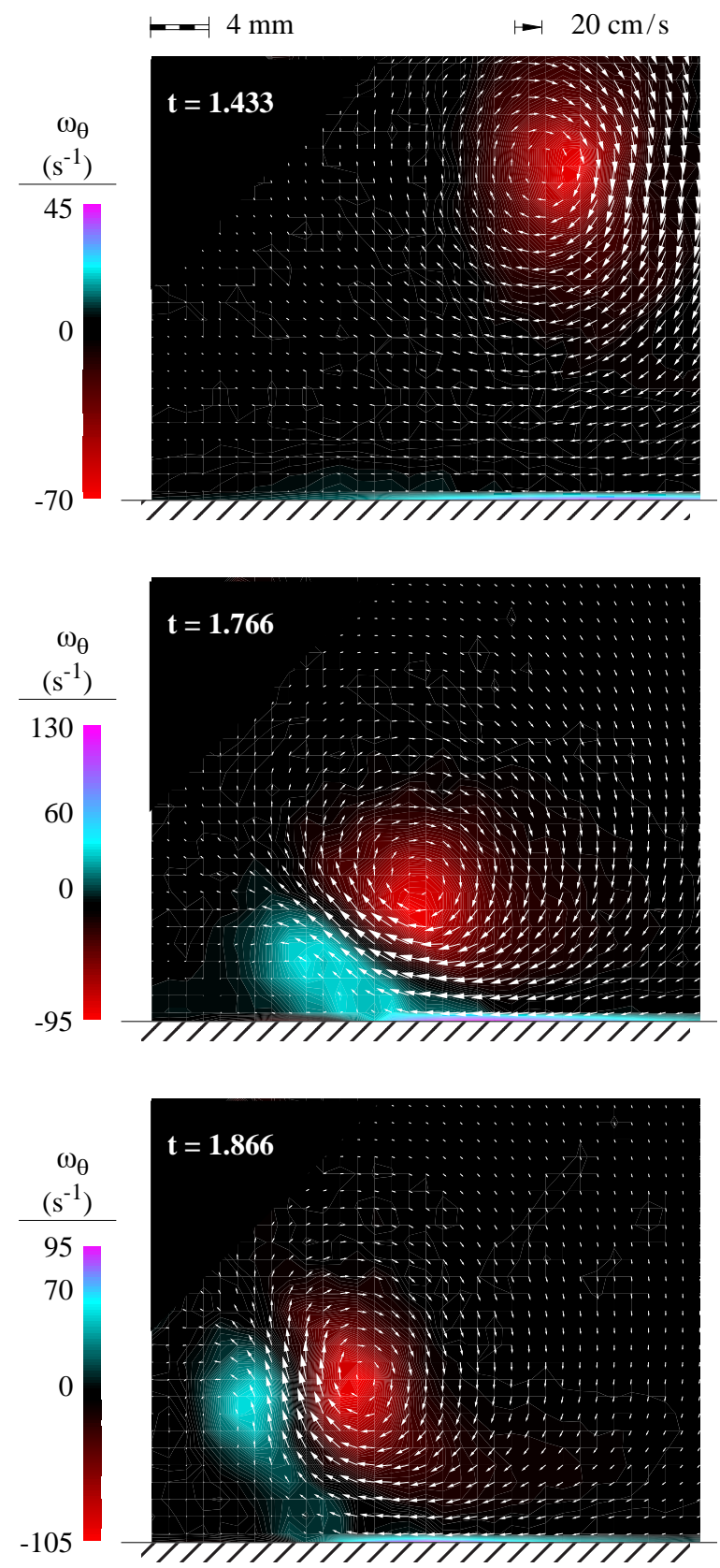

Fig. 9. Velocity and vorticity details of the ring/wall interaction measured using MTV. Only the left-half of the flow field is shown. 
The latter advantage of MTV offers a unique capability for velocimetry in highly three-dimensional flows, which we demonstrate in an application to a forced wake. We have previously reported that forcing a low Reynolds number 2-D wake downstream of a splitter plate can lead to a highly three-dimensional flow and a large increase in mixing (MacKinnon \& Koochesfahani 1993, Koochesfahani, et al. 1994). Figure 10 illustrates preliminary instantaneous velocity vectors (v, w components) in the cross-stream (y-z) plane at three different instances during the perturbation period at a downstream station $\mathrm{x}=13 \mathrm{~cm}$ from the splitter plate. The estimated streamwise vorticity fields at two of those instances are included in Figure 11. The region shown in these figures covers an area $1.8 \mathrm{~cm} \times 4 \mathrm{~cm}$. As before, except for remapping onto a regular grid, these are raw data. It is important to recognize that the mean streamwise flow direction is actually out of the page in this flow field. The high degree of three-dimensionality and the spatial structure of the streamwise vorticity are apparent. The maximum cross-stream velocity in Figure 10 is about $40 \%$ of the wake freestream velocity.

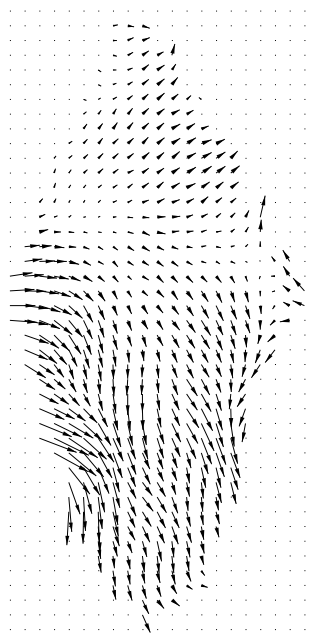

$t / T=0.2$

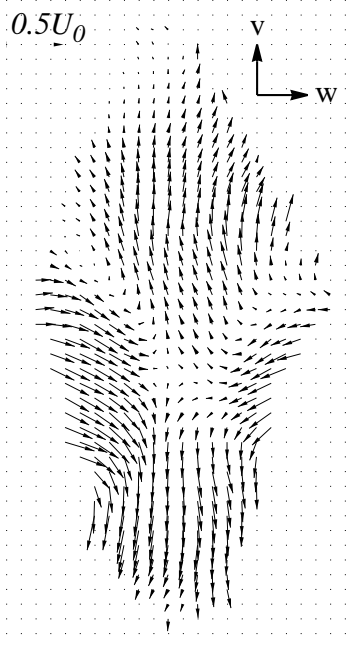

$t / T=0.4$

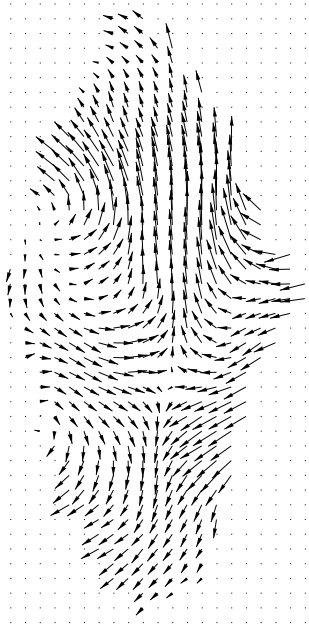

$t / T=0.6$

Fig. 10. Raw instantaneous MTV velocity vectors of the cross-stream flow in a forced wake at three different instances in the forcing cycle ( $T$ is the forcing period). Note that the mean flow is out of the page.

\section{Passive Scalar Mixing Dynamics}

In the non-reacting (passive scalar) implementation, the alcohol, $\mathrm{CD}$, and lumophore are premixed in one stream, and the other mixing stream is for example pure water or contains an alcohol solution. The Lagrangian evolution of the scalar mixing field is then monitored over the luminescence lifetime. An example of this 

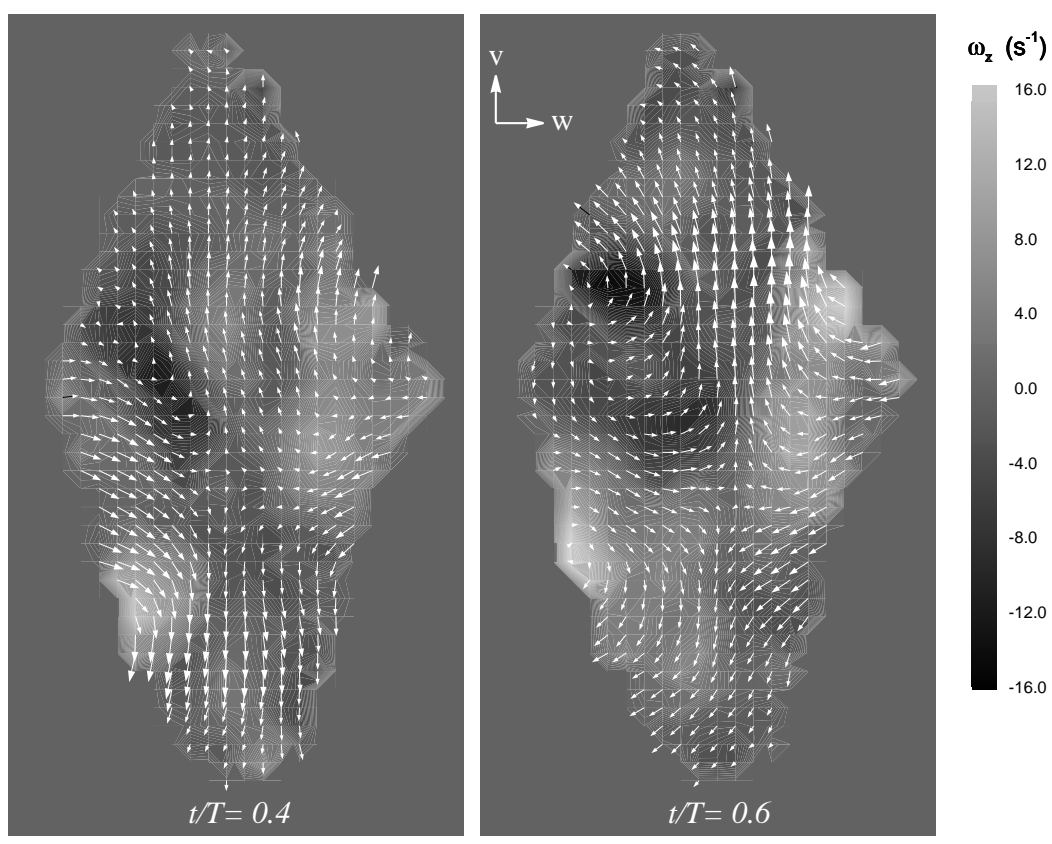

Fig. 11. Instantaneous MTV velocity vectors and streamwise vorticity of the cross-stream flow in a forced wake.

implementation is shown in Figure 12. A vortex ring's fluid is premixed with the alcohol/CD/lumophore solution, and the ambient fluid contains only the alcohol solution. The UV laser is arranged to illuminate a series of parallel "bands" in the flow. Figure 12 shows the molecularly-tagged patches of the vortex ring fluid at the initial time ( $20 \mu \mathrm{s}$ after laser firing), marked light grey, and the evolution of the same patches 9 ms later (marked dark grey). The velocity vectors at the corners of the patches are determined using the same direct spatial correlation technique described earlier. Figures 13(a,b) show the velocity vectors in the laboratory frame and the vortex frame, respectively.

Even though the vortex ring example just shown has a rather simple concentration field (i.e. uniform within the tagged patches), it does highlight the potential of a molecular tagging approach for simultaneous concentration (from the first image) and velocity (using image pairs as in Figure 12) measurements in more complex and turbulent flows. In this case the spatially non-uniform scalar concentration field typical of most turbulent flows can be used as a natural source of the required luminescence intensity variation described earlier. Where this can be accomplished, a much simpler optical arrangement with a laser sheet can replace the more involved technique of "writing" a prescribed laser pattern into the flow (e.g. the usual grid pattern). An example is given in Figure 14, illustrating the vortex ring of a starting jet illuminated by a pair of crossing laser sheets. The placement of the chemical components is the same as that in the example of Figure 12. 


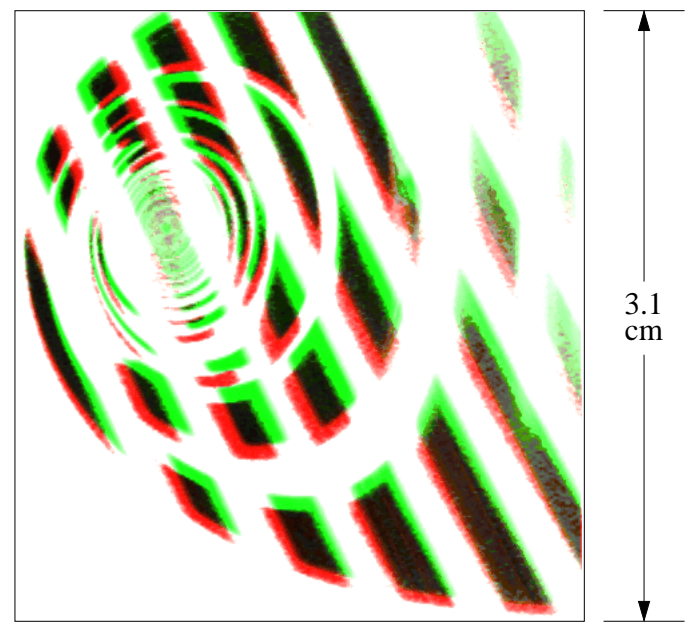

Fig. 12. Lagrangian evolution of molecularly tagged fluid patches in a vortex ring. Only the left half of a downward-moving ring is shown. The patches are regions imaged $20 \mu \mathrm{s}$ after laser firing; the $\square$ patches are the same regions $9 \mathrm{~ms}$ later.

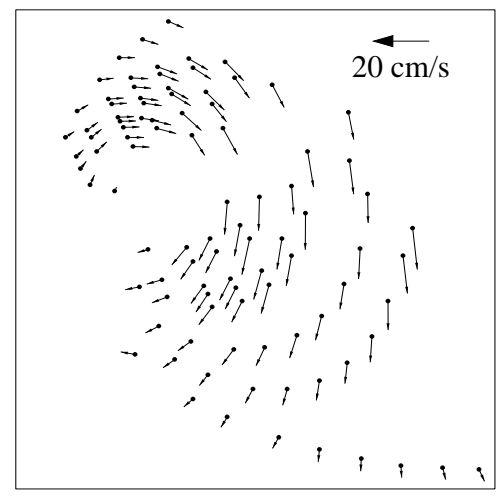

(a)

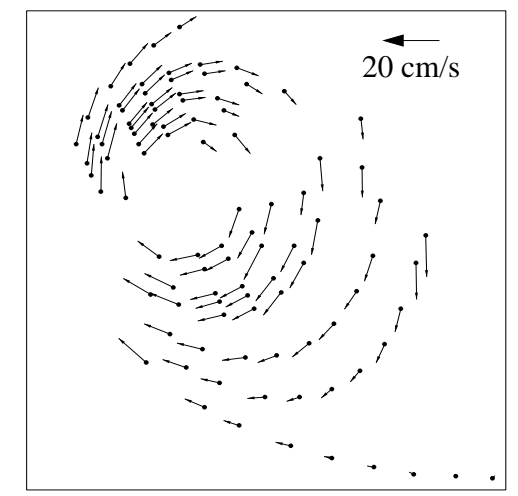

(b)

Fig. 13. (a) Velocity vectors estimated using the corners of the patches in Figure 12. (b) The velocity field of (a) with the core convection velocity subtracted.

Figure 14(a) is the first image of the tagged flow acquired $20 \mu$ s after the laser firing; the second image, not shown here, is acquired $6 \mathrm{~ms}$ later. In a manner similar to that already described, the Lagrangian displacement of small regions (source windows) in the first image can be obtained using spatial correlation with "roam windows" from the second image. One example of a suitable source window is illustrated in Figure 14(b) along with the roam window from the second image inFigure 14(c); the dashed black square in the roam window indicates the initial location of the source window. The resulting correlation coefficient field indicates 


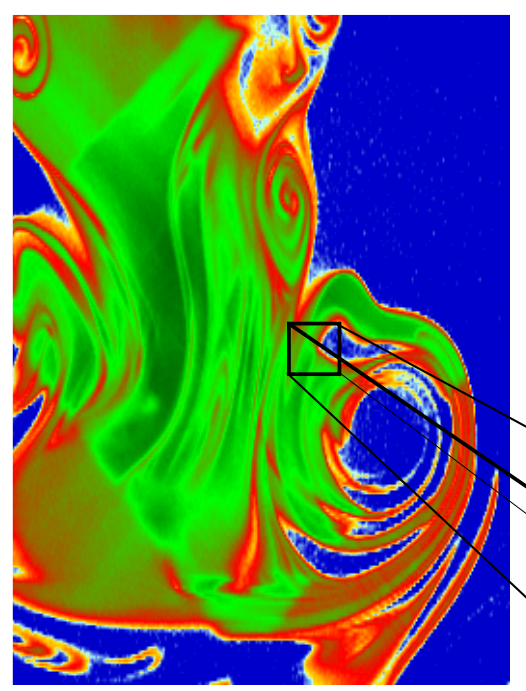

(a)

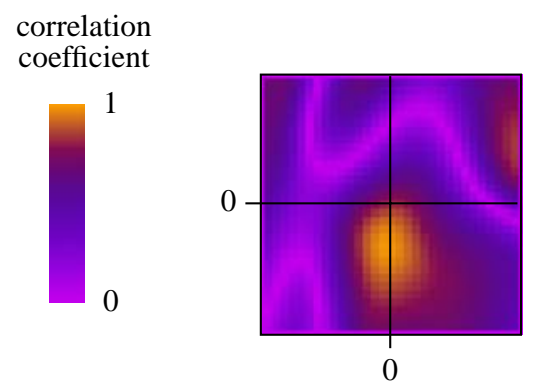

(d)

pixel

intensity

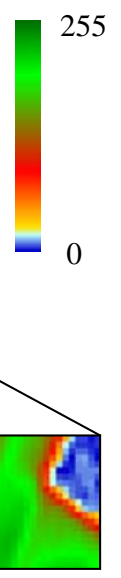

(b)

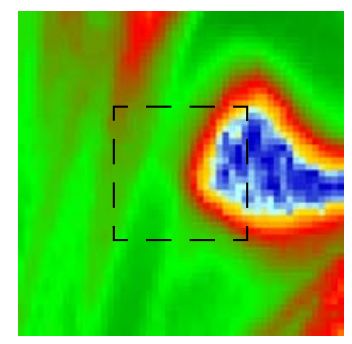

(c)

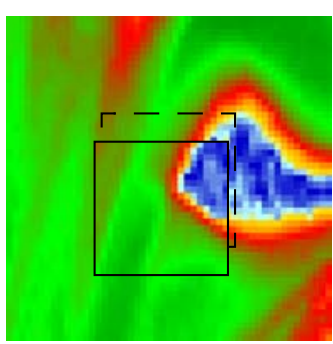

(e)

Fig. 14. Correlation technique applied to experimental data from a mixing jet flow field. (a) Earlier image. (b) $21 \times 21$ pixel source window from (a). (c) 51 x 51 pixel roam window from the second image (not shown here). (d) correlation coefficient field. (e) superposition of source window over roam window.

a well-defined peak whose pixel coordinates are determined to be $(\Delta x, \Delta y)=(-0.9$, -5.3). Figure 14(e) shows the superposition of (b) over (c), after (b) has been moved to the location of the peak correlation (the solid black square).

It is implicitly understood that the correlation procedure applied to continuously distributed luminescence fields such as Figure 14(a) will yield accurate displacement information in two directions only if sufficient intensity gradients are present within the selected source windows. In the areas of the flow where this requirement is not satisfied, a non-uniform laser illumination can be used instead. The example of Figure 14 again highlights the potential for simultaneous concentration 
(from the first image) and velocity (using image pairs) measurements. Similar to the discussion earlier in Section 2.3, in cases where monitoring the evolution of the passive scalar field over very long time periods is desired, a much longer lifetime tracer such as caged fluorescein should be used.

\section{Conclusions}

New developments in molecular tagging diagnostics for velocimetry (MTV) and the study of the Lagrangian evolution of scalar mixing fields have been described. The MTV approach is the molecular counterpart of PIV and as such offers certain advantages. In addition MTV tends to have much reduced sensitivity to out-ofplane motion contaminating in-plane velocity data. This aspect has been demonstrated in an application of MTV to the highly three-dimensional velocity field of a forced wake. The potential of the molecular tagging approach for studying the dynamic evolution of mixing interfaces and combined scalar/velocity measurements has also been demonstrated.

\section{Acknowledgments}

This work was supported in part by the Air Force Office of Scientific Research under grants F49620-92-J-0338 and F49620-95-1-0391, and the MRSEC Program of the National Science Foundation Award Number DMR-9400417.

\section{References}

Biage, M., Harris, S. R., Lempert, W. R., and Smits, A. J. 1996, Quantitative velocity measurements in turbulent Taylor-Couette flow by PHANTOMM flow tagging, Proceedings of the Eighth International Symposium on Applications of Laser Techniques to Fluid Mechanics, Lisbon, Portugal, July 8-11, 1996, 15.4.115.4.8.

Chu, C. C. and Liao, Y. Y. 1992, A quantitative study of the flow around an impulsively started circular cylinder, Exp. Fluids, vol. 13, 137-146.

Chu, C. C., Wang, C. T., and Hsieh, C. H. 1993, An experimental investigation of vortex motions near surfaces, Phys. Fluids A., vol. 5, no. 3, 662-676.

Cohn, R. K., Gendrich, C. P., Mackinnon, C. G., and Koochesfahani, M. M. 1995, Crossflow velocimetry measurements in a wake flow, Bull. Am. Phys. Soc., vol. 40, no. 12, 1962.

D'Arco, A., Charmet, J. C., and Cloitre, M. 1982, Nouvelle technique de marquage d'ecoulement par utilisation de molecules photochromes, Revue Phys. Appl., vol. 17, 89-93. 
Douglas, P., Enos, R. D., Azzopardi, B., and Hope, C. B. 1991, Characterisation of a photochromic triarylmethane dye sulphite and its applications to the visualisation of water flows, 7th Int. Topical Meeting on Photoacoustic and Photothermal Phenomena, The Netherlands, August, 1991.

Falco, R. E. and Chu, C. C. 1987, Measurement of two-dimensional fluid dynamic quantities using a photochromic grid tracing technique, SPIE, vol. 814, 706-710.

Falco, R. E. and Nocera, D. G. 1993, Quantitative multipoint measurements and visualization of dense solid-liquid flows using laser induced photochemical anemometry (LIPA), in Particulate Two-Phase Flow, Ed. M. C. Rocco; ButterworthHeinemann, 59-126.

Gendrich, C. P. and Koochesfahani, M. M. 1996, A spatial correlation technique for estimating velocity fields using Molecular Tagging Velocimetry (MTV), Exp. Fluids, vol. 22, no. 1, 67-77.

Gendrich, C. P., Koochesfahani, M. M., and Nocera, D. G. 1994, Analysis of molecular tagging velocimetry images for obtaining simultaneous multi-point velocity vectors, Bull. Am. Phys. Soc., vol. 39, no. 9, 1980.

Gendrich, C. P., Koochesfahani, M. M., and Nocera, D. G. 1996, Molecular tagging velocimetry and other novel applications of a new phosphorescent supramolecule, submitted to Exp. Fluids.

Harris, S. R., Lempert, W. R., Hersh, L., Burcham, C. L., Saville, A., Miles, R. B., Gee, K., and Haughland, R. P. 1996a, Quantitative measurements on internal circulation in droplets using flow tagging velocimetry, AIAA J., vol. 34, no. 3, 449454.

Harris, S. R., Miles, R. B., and Lempert, W. R. 1996b, Observations of fluid flow produced in a closed cylinder by a rotating lid using the PHANTOMM (Photo-Activated Non Intrusive Tracking of Molecular Motion) flow tagging technique, Proceedings of the Eighth International Symposium on Applications of Laser Techniques to Fluid Mechanics, Lisbon, Portugal, July 8-11, 1996, 15.3.115.3.9.

Hartmann, W. K., Gray, M. H. B., Ponce, A., and Nocera, D. G. 1996, Substrate induced phosphorescence from cyclodextrin - lumophore host-guest complexes, Inorg. Chim. Acta, vol. 243, 239.

Hilbert, H. S. and Falco, R. E. 1991, Measurements of flows during scavenging in a two-stroke engine, SAE Technical Paper 910671.

Hill, R. B. and Klewicki, J. C. 1995, Data reduction methods for flow tagging velocity measurements, Exp. Fluids, vol. 20, no. 3, 142-152.

Koochesfahani, M. M., Gendrich, C. P., and Nocera, D. G. 1993, A new technique for studying the Lagrangian evolution of mixing interfaces in water flows, Bull. Am. Phys. Soc., vol. 38, no. 12, 2287.

Koochesfahani, M. M., Beresh, S. J., and MacKinnon, C. G. 1994, Volumetric visualization of 3-D flow structure in a low Reynolds number forced wake, Bull. Am. Phys. Soc., vol. 39, no. 9, 1947. 
Lempert, W. R., Magee, K., Ronney, P., Gee, K. R., and Haughland, R. P. 1995, Flow tagging velocimetry in incompressible flow using photo-activated nonintrusive tracking of molecular motion (PHANTOMM), Exp. Fluids, vol. 18, 249-257.

Lu, L. J. and Smith, C. R. 1985, Image processing of hydrogen bubble visualization for determination of turbulence statistics and bursting characteristics, Exp. Fluids, vol. 3, 349-356.

MacKinnon, C. G. and Koochesfahani, M. M. 1993, Three-dimensional structure and mixing of a forced wake inside a confined channel, AIAA Paper 93-0658.

Miles, R., Cohen, C., Conners, J., Howard, P., Huang, S., Markovitz, E., and Russell, G. 1987, Velocity measurements by vibrational tagging and fluorescent probing of oxygen, Optics Letters, vol. 12, no. 11, 861-863.

Miles, R. B., Connors, J. J., Markovitz, E. C., Howard, P. J., and Roth, G. J. 1989, Instantaneous profiles and turbulence statistics of supersonic free shear layers by Raman excitation plus laser-induced electronic fluorescence (Relief) velocity tagging of oxygen, Exp. Fluids, vol. 8, 17-24.

Miles, R. B., Zhou, D., Zhang, B., Lempert, W. R., and She, Z. S. 1993, Fundamental turbulence measurements by Relief flow tagging, AIAA J., vol. 31, no. 3, 447-452.

Mortellaro, M. A. and Nocera, D. G. 1996, A turn-on for optical sensing, Chemtech, vol. 26, 17-23.

Ojha, M., Cobbold, R. S. C., Johnston, K. W., and Hummel, R. 1989, Pulsatile flow through constricted tubes: an experimental investigation using photochromic tracer methods, J. Fluid Mech., vol. 203, 173-197.

Ponce, A., Wong, P. A., Way, J. J. and Nocera, D. G. 1993, Intense phosphorescence triggered by alcohols upon formation of a cyclodextrin ternary complex, $J$. Physical Chem., vol. 97, 11137-11142.

Popovich, A. T. and Hummel, R. L. 1967, A new method for non-disturbing turbulent flow measurement very close to a wall, Chem. Eng. Soc., vol. 22, 21-25.

Stier, B. 1994, An investigation of fluid flow during the induction stroke of a water analog model of an IC engine using an innovative optical velocimetry concept -- LIPA, PhD thesis, Michigan State University, East Lansing, Michigan.

Stier, B., Koochesfahani, M. M., Nocera, D. G., and Schock, H. J. 1995, Molecular tagging velocimetry in gas phase flow, Bull. Am. Phys. Soc., vol. 40, no. 12, 1962.

Yurechko, V. N. and Ryazantsev, Yu. S. 1991, Fluid motion investigation by the photochromic flow visualization technique, Exp. Thermal and Fluid Sci., vol. 4, 273-288. 